\title{
DIRECT MAPPING OVSF-BASED TRANSMISSION SCHEME FOR UNDERWATER ACOUSTIC MULTIMEDIA COMMUNICATION
}

\author{
Chin-Feng Lin \\ Department of Electrical Engineering, National Taiwan Ocean University, Keelung, Taiwan, R.O.C, \\ Icf1024@mail.ntou.edu.tw \\ Jiang-Yao Chen \\ Department of Electrical Engineering, National Taiwan Ocean University, Keelung, Taiwan, R.O.C \\ Ya-Ju Yu \\ Department of Electrical Engineering, National Taiwan Ocean University, Keelung, Taiwan, R.O.C \\ Jung-Ting Yan \\ Department of Electrical Engineering, National Taiwan Ocean University, Keelung, Taiwan, R.O.C \\ Shung-Hyung Chang \\ Department of Microelectronic Engineering, National Kaohsiung Marine University, Kaohsiung, Taiwan, R.O.C.
}

Follow this and additional works at: https://jmstt.ntou.edu.tw/journal

Part of the Electrical and Computer Engineering Commons

\author{
Recommended Citation \\ Lin, Chin-Feng; Chen, Jiang-Yao; Yu, Ya-Ju; Yan, Jung-Ting; and Chang, Shung-Hyung (2010) "DIRECT MAPPING OVSF- \\ BASED TRANSMISSION SCHEME FOR UNDERWATER ACOUSTIC MULTIMEDIA COMMUNICATION," Journal of Marine \\ Science and Technology. Vol. 18: Iss. 3, Article 11. \\ DOI: $10.51400 / 2709-6998.1887$ \\ Available at: https://jmstt.ntou.edu.tw/journal/vol18/iss3/11 \\ This Research Article is brought to you for free and open access by Journal of Marine Science and Technology. It has been \\ accepted for inclusion in Journal of Marine Science and Technology by an authorized editor of Journal of Marine Science and \\ Technology.
}


DIRECT MAPPING OVSF-BASED TRANSMISSION SCHEME FOR UNDERWATER ACOUSTIC MULTIMEDIA COMMUNICATION

Acknowledgements

The authors acknowledge the support of the grant from the National science Council of Taiwan NSC 93-2218-E-019-024 as well as 96-2221-E-002-016 and the valuable comments of the reviewers. 


\title{
DIRECT MAPPING OVSF-BASED TRANSMISSION SCHEME FOR UNDERWATER ACOUSTIC MULTIMEDIA COMMUNICATION
}

\author{
Chin-Feng Lin*, Jiang-Yao Chen*, Ya-Ju Yu*, Jung-Ting Yan*, and Shung-Hyung Chang**
}

Key words: OVSF, power assignment, underwater acoustic multimedia communication.

\begin{abstract}
Underwater communication networks are currently being developed for use in underwater control systems, underwater telemetry systems, and underwater image transmission systems. The transmission bandwidth and transmission rates of an underwater communication system are low, and its transmission delay is high. In this paper, we propose a direct mapping orthogonal variable spreading factor (OVSF)-based transmission system for underwater acoustic multimedia communication. An important feature of this transport architecture is that a power assignment mechanism, an OVSF scheme, a direct mapping strategy, an unequal error protection scheme, and adaptive modulation are employed for transmitting messages that can tolerate various bit error rates. For confirming the effectiveness of this transport architecture, we perform a simulation using measured data of a G.729 audio signal and a JPEG2000 image signal. The simulation results show that the proposed system can achieve maximum transmission data rates or minimum transmission power. In addition, the OVSFbased transport architecture is a feasible platform in underwater acoustic multimedia communication.
\end{abstract}

\section{INTRODUCTION}

A major part of the earth's surface is covered by the ocean. Deep oceans are popularly known as inner space. Humans have often enthusiastically explored this inner space in order to gain a better understanding of the underwater world. A popular and interesting area of research is underwater acoustic communication [1-3, 5-6, 8-9, 11, 15-18, 20-21, 24]. At present, underwater acoustic transmission system can be used to

Paper submitted 10/29/08; revised 06/29/09; accepted 06/30/09. Author for correspondence: Chin-Feng Lin (e-mail: lcf1024@mail.ntou.edu.tw).

*Department of Electrical Engineering, National Taiwan Ocean University, Keelung, Taiwan, R.O.C.

**Department of Microelectronic Engineering, National Kaohsiung Marine University, Kaohsiung, Taiwan, R.O.C. issue command/control instructions in underwater systems, to measure transmission data of underwater measurement instruments such as sonar systems, and to transmit underwater images. It is similar to land communication using multiple access schemes. Examples of multiple access schemes include frequency division multiple access (FDMA), time division multiple access (TDMA), spread spectrum technique, and orthogonal frequency division multiplexing (OFDM). However, due to a harsh underwater environment, underwater communication faces some unique problems such as limited bandwidth, high and variable propagation delays, high bit error rates (BER), and severe frequency-selective distortion caused by multipath propagation. Spread spectrum communication is considered as a candidate technique for use in future mobile underwater acoustic networks. Its advantages include efficient use of bandwidth, flexible guard band as compared to that in FDMA, and less stringent synchronization requirements as compared to those in TDMA. In the past, various studies have been conducted to investigate the technologies used in underwater acoustic communication systems. Authors proposed an underwater adaptive-array receiver structure in which direct-sequence code division multiple access (DS-CDMA) and spatial diversity combining are used for achieving reliable low-data rate multiuser communication in an asynchronous shallow-water network [24]. In [5], the authors found that in underwater acoustic networks, the performance of a direct-sequence spread spectrum system is better than frequency-hopping spread-spectrum system in terms of the transmission BER. In [9], the authors use DS-CDMA and multi-carrier CDMA to achieve reliable multiuser communication in asynchronous shallow-water acoustic networks. In DS-CDMA, spread data are transmitted at a single carrier frequency. In contrast, in MC-CDMA, a set of carrier frequencies is used to achieve frequency diversity. In [9], the authors found that for low signal-to-noise ratios (SNRs), the performance of the DS-CDMA system was better than that of the MC-CDMA system and for SNR values higher than $20 \mathrm{~dB}$, the MC-CDMA system outperformed the DS-CDMA system due to the frequency diversity of the former system. In [21], the authors proposed a multichannel detection method and an efficient channel-estimation-based multiuser detection method for wideband underwater acoustic CDMA communication. In 


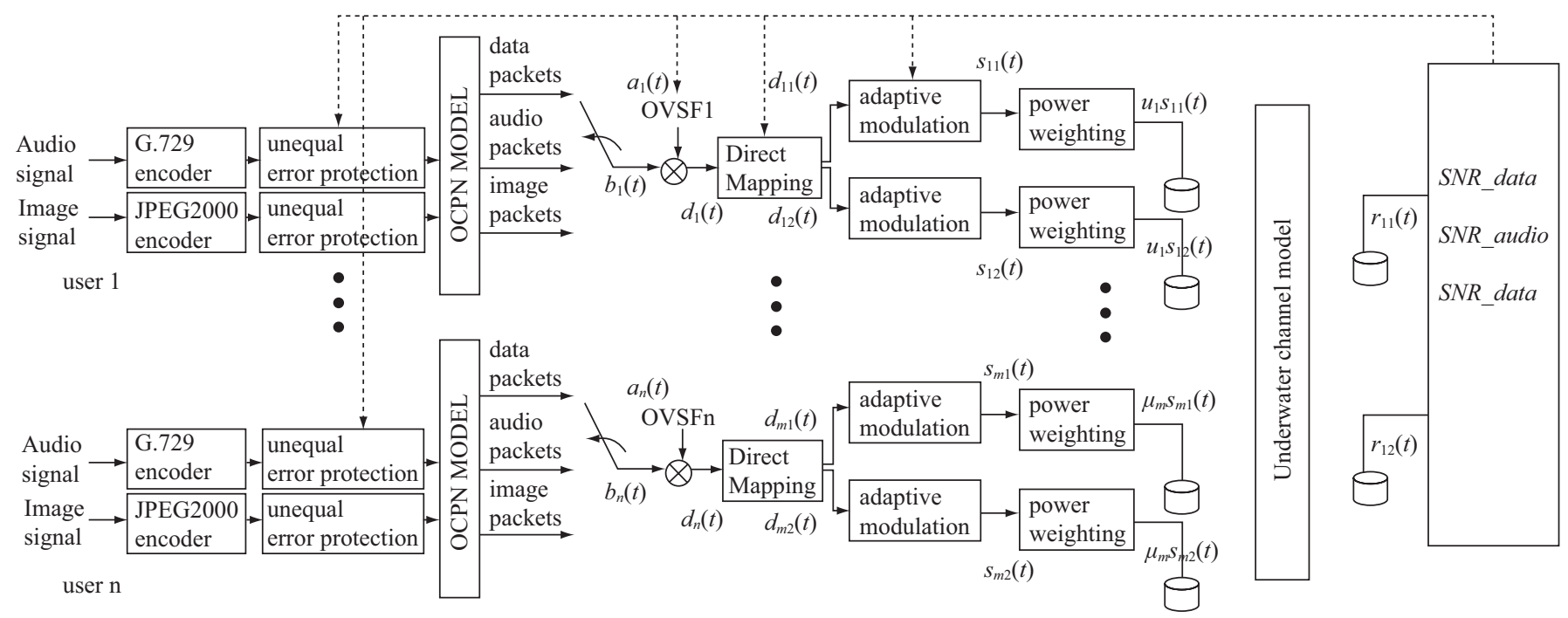

Fig. 1. Proposed OVSF-based underwater acoustic transmission system.

[16], the authors developed a dynamic multiple access protocol for different underwater acoustic network architectures and traffic scenarios; this protocol efficiently shares scarce underwater channel bandwidth by fully leveraging CDMA medium access properties. They also implemented a novel closed-loop distributed algorithm to jointly set the optimal values of transmit power and code length that combat for the near-far effect. In previous studies [4, 10, 11-15], we examined wireless multi-media communication, mobile telemedicine systems, and underwater acoustic multi-media communication. For example, in [15], we studied a single input single output (SISO) OFDM-based transmission scheme for underwater acoustic multimedia communication. Further, in [11], we studied a transmission scheme based on SISO orthogonal variable spreading factor (OVSF) codes for use in underwater acoustic multimedia communication. In this study, we extend this previously work [11], and develop an underwater acoustic multimedia transmission system in which the OVSF, a power assignment mechanism, an adaptive modulation scheme, direct mapping (DM) and an unequal error protection scheme are employed for transmitting messages with low error probability and at low power. We jointly set the transmit power, code length, modulation type, and coding type for underwater multimedia communication and to combat for the near far effect. In addition, we show that the proposed underwater acoustic multimedia communication system can achieve maximum transmission data rates or minimum transmission power.

\section{DIRECT MAPPING OVSF-BASED TRANSMISSION SCHEME FOR UNDERWATER ACOUSTIC MULTIMEDIA COMMUNICATION}

OVSF-based transmission is one of the various multiple access techniques employed in an underwater acoustic communication system. An advantage of such a system is that it can facilitate robust data transmission. The transmission architecture of the proposed system is shown in Fig. 1. From this figure, we can observe that the proposed system can effectively deal with various types of signals in an underwater environment. When users play multimedia in the receiver of the underwater acoustic communication system, various multimedia objects are synchronized. The time dependent relations among various objects as well as their play throughput and transmission data rates for various objects are estimated. A model called the object-composition Petri-net (OCPN) [25] can describe the temporal relationships among the various aspects of multimedia information, such as its type, size, throughput requirements, and duration for which it is presented in the receiver. Such aspects can then be delivered by the proposed underwater acoustic multimedia communication system. Usually, the quality of service (QoS) requirements for various messages in a multimedia system are different. Here, we assume that the acceptable BERs for audio, and image packets are $10^{-3}$, and $10^{-4}$, respectively. A $1500 \mathrm{kbits}$ test image signal is compressed by a Joint Photographic Experts Group 2000 (JPEG2000) encoder to produce a $100 \mathrm{kbits}$ image bit stream. Further, a 1330 kbits test audio signal is compressed by a G.729 encoder to produce a 96 kbits audio bit stream. These image, and audio bit streams are introduced in the OCPN model. Two types of packets-image and audio packets-are used in our OVSF-based underwater acoustic communication system for transmitting audio, and image bit streams, respectively. The total number of audio and image channels can easily be estimated by the OCPN model. OVSF codes are applied in spread data to combat channel fading and achieve the required QoS for underwater acoustic multimedia transmission. There is only one code in OVSF with spreading factor 1 , and the first OVSF code is 1 . The second OVSF code $(1,-1)$ is generated from the first OVSF code, and the spreading factor of the second code is 2 . In addition, the $i$ th OVSF 
code $C$ with an spreading factor of $2^{i-1}$, and the $i$ th OVSF code set having $2^{i-1}$ OVSF codes can be generated as

$$
C_{i}=\left\{\begin{array}{ccc}
C_{i-1} C_{i-1} & \text { if } & x_{i}=0 \\
C_{i-1}\left(-C_{i-1}\right) & \text { if } & x_{i}=1
\end{array}\right.
$$

In our proposed system, we use the carrier sense multiple access/collision avoidance (CSMA/CA) protocol [7], and the lengths of the OVSF code $c(t)$ are 8, 16, 32, and 64 for various packets. CSMA/CA belongs to a class of protocols called multiple access schemes. In CSMA/CA, a station wanting to transmit data must first listen to the shared channel for a predetermined amount of time so as to check for any activity on the channel. If the channel is sensed as "idle," that the station is permitted to transmit. If the channel is sensed as "busy," the station has to defer its transmission. The baseband transmission signal $d_{m}(t)$ obtained using the $m$ th OVSF code for the $m$ th user is expressed as

$$
d_{m}(t)=a_{m}(t) b_{m}(t)
$$

Here, $b_{m}(t)$ is the data signal, which consists of a sequence of rectangular pulses of duration $T$, for the $m$ th user, and $b_{m}(t)$ is the $m$ th OVSF spreading sequence for the $m$ th user. The output sequence of $d_{m}(t)$ is $\left(d_{m 1}, d_{m 2}, d_{m 3}, d_{m 4}, \ldots\right)$, and then, it is subjected to DM [26]. DM is a transmission mechanism in the IEEE $802.11 \mathrm{n}$ standard, and it assigns the sequence $\left(d_{m 1}\right.$, $\left.d_{m 2}, d_{m 3}, d_{m 4}, \ldots\right)$ sequence to $\mathrm{N}$ parallel units. Further, a serial-to-parallel converter also splits this sequence, $d_{m 1}, d_{m 2}, d_{m 3}$, $d_{m 4}$, into $N$ branches. Thus, we describe $d_{m 1}(t)=d_{1}(m t), m=1$, $3,5, \ldots$, and $d_{m 2}(t)=d_{1}(m t), m=2,4,6, \ldots$ The first transducer is used to transmit $s_{m 1}(t)$, the modulation chip sequence of $d_{m 1}(t)$. Similarly, the second transducer is used to transmit $s_{m 2}(t)$, the modulation chip sequence of $d_{m 2}(t)$. DM can achieve high transmission data rates for underwater acoustic multimedia communication. The received $l$ th hydrophone signal for $m$ th user is expressed as

$$
\gamma_{m l}(t)=\sum_{n=1}^{N} u_{m} P S_{m n}(t) * h_{m n l}(t)+n_{m l}(t)
$$

Here, constant $P$ is the transmission power of the transmitter; $\mu_{m}$ is the transmission power weighting factor, and $0<\mu_{m} \leq 1$. Further, $h_{i l}$ is the channel impulse response of the $i$ th transducer and $l$ th hydrophones; We assume perfect channel estimation, and $n_{m l}(t)$ is the additive white Gaussian noise (AWGN) for the $m$ th user, obtained by the receiver using the $l$ th hydrophones. L is the total number of hydrophones in the receiver. The SNR obtained by receiver for the $m$ th user is given by

$$
S N R_{m}=\frac{\sum_{l=1}^{L} E\left\{r_{m l}^{2}(t)\right\}}{\sum_{l=1}^{L} E\left\{n_{m l}^{2}(t)\right\}}
$$

Thus, the decision on the output is

$$
\begin{aligned}
& D_{m}(t)=\sum_{l=1}^{L} r_{m l}(t) a(t) \\
& \hat{b}_{m}(t)=\operatorname{dec}\left\{\mathrm{D}_{m}(t)\right\}
\end{aligned}
$$

For $D_{m}(t)$, the receiver makes a decision according to specified thresholds. We summarize our power assignment algorithm as follows:

Step 1: On the basis of the output information of the OCPN model, obtain throughputs of audio and image messages for underwater transmission.

Step 2: Select an appropriate unequal error protection parameter and modulation mode to satisfy the requirements for an underwater system.

Step 3: Assign the transmission power weighting factor $\mu, 0<$ $\mu \leq 1$, for audio or image packets.

Step 4: Measure the received SNR values in the cases of audio or image packets.

Step 5: If the measured SNR of the received signal is larger than the threshold SNR that can yield the required $\mathrm{BER}$, update the transmission power weighting factor to $\mu=\mu-\Delta$ and go to Step 4. Otherwise, go to Step 6 .

Step 6: Increase the transmission power weighting factor to $\mu=\mu+\Delta$. If $\mu>1$, reselect both the unequal error protection parameter and the modulation mode, and go to Step 3. If $\mu \leq 1$, go to Step 4 and repeat the remaining steps.

The value of parameter $\Delta$ depends on the variation in channel fading. The greater the variation in channel fading, the larger is the value of $\Delta$. In addition, the smaller the $\Delta$ variation, the larger is the amount of power saved. The lengths of the OVSF codes for audio, and video packets_LC_a and LC_v-are 8, 16, 32, and 64. The channel coding rates are obtained using $1 / 2(561,753)$ and $1 / 3(557,663,771)$ convolution codes. The possible length of OVSF codes, modulation type, and channel coding for audio, video, and data packets are (64, 1/2, BPSK), (64, 1/2, QPSK), (32, 1/2, BPSK), (32, 1/2, QPSK), (16, 1/2, BPSK), (16, 1/2, QPSK), (8, 1/2, BPSK), (8, 1/2, QPSK), (64, 1/3, BPSK), (64, 1/3, QPSK), (32, 1/3, BPSK), (32, 1/3, QPSK), (16, 1/3, BPSK), (16, 1/3, QPSK), (8, $1 / 3$, BPSK), and (8, 1/3, QPSK). The initial power for audio, video, and data packets is $1 / 30$, and the maximum power is 1 .

\section{SIMULATION}

We performed a simulation to demonstrate the functionality of the proposed OVSF-based underwater transmission system. In this simulation, we used adaptive modulation, DM, CSMA/ CA, OVSF codes, power assignment algorithm, and the unequal error protection scheme. We used the underwater chan- 


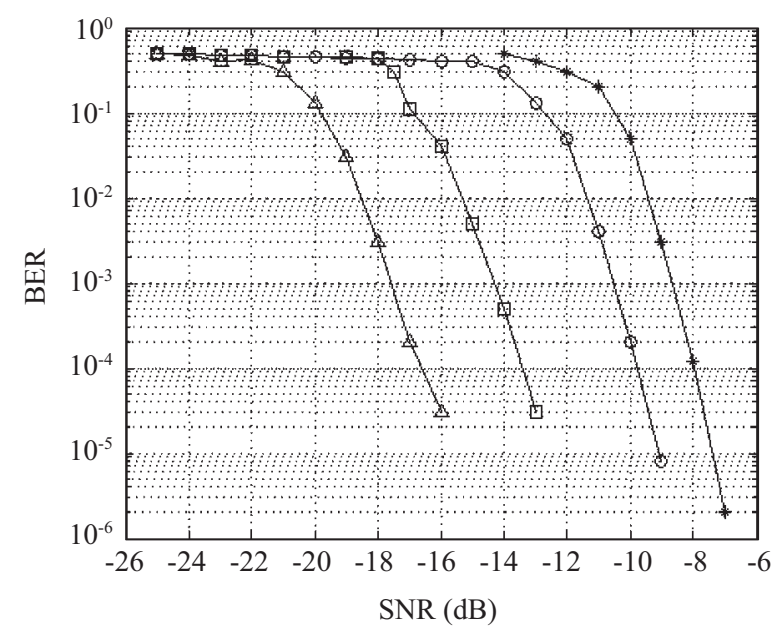

Fig. 2. Bit error rate performance of proposed OVSF-based underwater acoustic transmission system with $1 / 2(561,753)$ coding and BPSK modulation. (*: spreading factor $(\mathrm{SF})=8$, O: spreading factor $(\mathrm{SF})=16, \square$ : spreading factor $(\mathrm{SF})=32, \triangle$ : spreading factor $(\mathrm{SF})=64)$

nel model developed by Zhang et al. [26] in this simulation. In this model, the transmission range is $1000 \mathrm{~m}$, the frequency of a carrier wave is $11.5 \mathrm{kHz}$, and the bandwidth is 3.90625 $\mathrm{kHz}$. The transmitter contains two transducers and the receiver contains two hydrophones. The BER performance of the proposed underwater transmission system is shown in Fig. 2. We use BPSK modulation, $K=91 / 2(561,753)$ and $1 / 3$ $(557,663,771)$ convolution codes with soft decoding [23], and a $2 \times 2$ DM strategy for the transmission of audio and image packets. The spreading factor for the OVSF codes are 8 , 16,32 , and 64 . The greater the length of spreading codes, the lower is the transmission BER. The transmission power weighting for the proposed system in the case of BERs of $10^{-3}$, and $10^{-4}$ for audio and image packets, respectively, under different noise conditions is shown in Fig. 3 as a function of the AWGN $\left(N_{o}\right)$. Here, the length of OVSF code is 8 . From Fig. 3, we can observe that the higher the noise, the higher is the transmission power. Further, less restrictions on the transmission BER result in low transmission power. The transmission power weighting of audio signals is smaller than that of image signals. Further, the transmission power weighting of $1 / 2$ convolution codes is larger than that of $1 / 3$ convolution codes. We consider No $=-10 \mathrm{~dB}$. The lengths of OVSF codes are $8,16,32$, and 64 . Smaller length of OVSF codes result in low transmission power. Tables 1 and 2 show the descend power and transmission data rates for various lengths of OVSF codes, modulation types, and channel coding types for audio and image signals, respectively. Here, No is $-10 \mathrm{~dB}$. The maximum transmission data rates is 976 bits for the bandwidth of $3.90625 \mathrm{kHz}$, and the maximum descend power is $80 \%$. The descend power is defined as

$$
\frac{R_{b}-R_{b} \times u_{b}}{R_{b}} \times 100 \%
$$

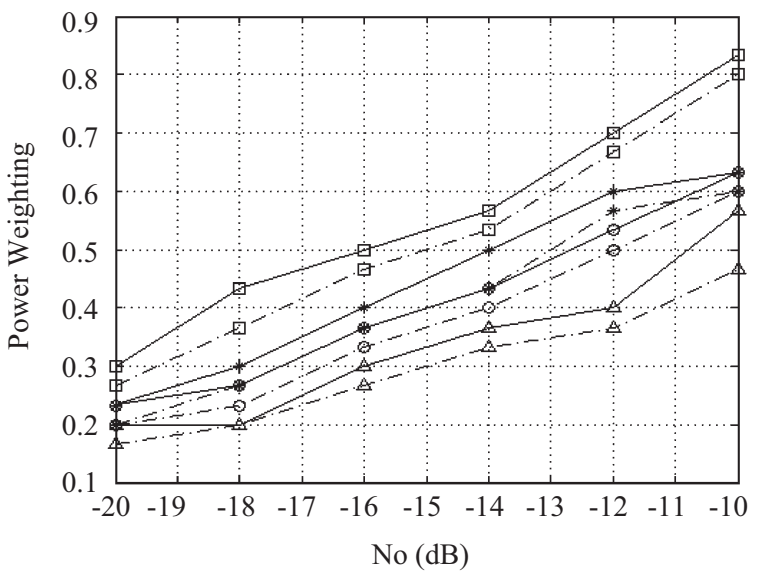

Fig. 3. Transmission power weighting performance of proposed OVSFbased underwater acoustic transmission system with $\mathrm{OVSF}=8$. ( $\triangle$, dotted line: $1 / 3$ convolution, BPSK, audio; $O$, dotted line: $1 / 3$ convolution, QPSK, audio; *, dotted line: 1/2 convolution, BPSK, audio; $\square$, dotted line: $1 / 2$ convolution, QPSK, audio; $\triangle$, line: $1 / 3$ convolution, BPSK, image; $O$, line: $1 / 3$ convolution, QPSK, image; *, line: $1 / 2$ convolution, BPSK, image; $\square$, line: $1 / 2$ convolution, QPSK, image)

Table 1. shows the descend power and transmission data rates for various the length of OVSF codes, modulation types, and channel coding types for audio signals.

\begin{tabular}{|c|c|c|c|c|c|}
\hline $\begin{array}{c}\text { the length of } \\
\text { OVSF code }\end{array}$ & modulation & $\begin{array}{c}\text { convolution } \\
\text { code }\end{array}$ & $\begin{array}{c}\text { transmission } \\
\text { data rates } \\
\text { (bits) }\end{array}$ & $\begin{array}{c}\text { power } \\
\text { weighting }\end{array}$ & $\begin{array}{c}\text { descend } \\
\text { power(\%) }\end{array}$ \\
\hline 8 & BPSK & $1 / 2$ & 488 & $18 / 30$ & 40 \\
\hline 8 & QPSK & $1 / 2$ & 976 & $25 / 30$ & 16.67 \\
\hline 8 & BPSK & $1 / 3$ & 324 & $14 / 30$ & 53.3 \\
\hline 8 & QPSK & $1 / 3$ & 648 & $18 / 30$ & 40 \\
\hline 16 & BPSK & $1 / 2$ & 244 & $14 / 30$ & 53.3 \\
\hline 16 & QPSK & $1 / 2$ & 488 & $18 / 30$ & 40 \\
\hline 16 & BPSK & $1 / 3$ & 162 & $11 / 30$ & 63.3 \\
\hline 16 & QPSK & $1 / 3$ & 324 & $14 / 30$ & 52.72 \\
\hline 32 & BPSK & $1 / 2$ & 122 & $9 / 30$ & 70 \\
\hline 32 & QPSK & $1 / 2$ & 244 & $13 / 30$ & 56.67 \\
\hline 32 & BPSK & $1 / 3$ & 80 & $7 / 30$ & 76.67 \\
\hline 32 & QPSK & $1 / 3$ & 162 & $11 / 30$ & 63.3 \\
\hline 64 & BPSK & $1 / 2$ & 60 & $7 / 30$ & 76.67 \\
\hline 64 & QPSK & $1 / 2$ & 120 & $9 / 30$ & 70 \\
\hline 64 & BPSK & $1 / 3$ & 40 & $6 / 30$ & 80 \\
\hline 64 & QPSK & $1 / 3$ & 80 & $7 / 30$ & 76.67 \\
\hline
\end{tabular}

Where $R_{b}$ and $\mu_{b}$ are the transmission data rate and the transmission power weighting, respectively, for audio or image signals. From Tables 1 and 2, we observe that the proposed system can achieve maximum transmission data rates or minimum transmission power. Figure 5 shows the performance of a G.729 audio signal in the proposed underwater transmission system with the power assignment mechanism. The mean square error (MSE) of the original and received 
Table 2. shows the descend power and transmission data rates for various the length of OVSF codes, modulation types, and channel coding types for image signals.

\begin{tabular}{|c|c|c|c|c|c|}
\hline $\begin{array}{l}\text { the length of } \\
\text { OVSF code }\end{array}$ & modulation & $\begin{array}{l}\text { convolution } \\
\text { code }\end{array}$ & $\begin{array}{c}\text { transmission } \\
\text { data rates } \\
\text { (bits) }\end{array}$ & $\begin{array}{c}\text { power } \\
\text { weighting }\end{array}$ & $\begin{array}{c}\text { descend } \\
\text { power }(\%)\end{array}$ \\
\hline 8 & BPSK & $1 / 2$ & 488 & $19 / 30$ & 36.67 \\
\hline 8 & QPSK & $1 / 2$ & 976 & $26 / 30$ & 13.33 \\
\hline 8 & BPSK & $1 / 3$ & 324 & $17 / 30$ & 43.3 \\
\hline 8 & QPSK & $1 / 3$ & 648 & $19 / 30$ & 36.67 \\
\hline 16 & BPSK & $1 / 2$ & 244 & $15 / 30$ & 50 \\
\hline 16 & QPSK & $1 / 2$ & 488 & $19 / 30$ & 36.67 \\
\hline 16 & BPSK & $1 / 3$ & 162 & $12 / 30$ & 60 \\
\hline 16 & QPSK & $1 / 3$ & 324 & $15 / 30$ & 49.38 \\
\hline 32 & BPSK & $1 / 2$ & 122 & $10 / 30$ & 66.67 \\
\hline 32 & QPSK & $1 / 2$ & 244 & $14 / 30$ & 53.33 \\
\hline 32 & BPSK & $1 / 3$ & 80 & $8 / 30$ & 73.33 \\
\hline 32 & QPSK & $1 / 3$ & 162 & $12 / 30$ & 60 \\
\hline 64 & BPSK & $1 / 2$ & 60 & $8 / 30$ & 73.33 \\
\hline 64 & QPSK & $1 / 2$ & 120 & $11 / 30$ & 63.33 \\
\hline 64 & BPSK & $1 / 3$ & 40 & $7 / 30$ & 76.67 \\
\hline 64 & QPSK & $1 / 3$ & 80 & $8 / 30$ & 73.33 \\
\hline
\end{tabular}

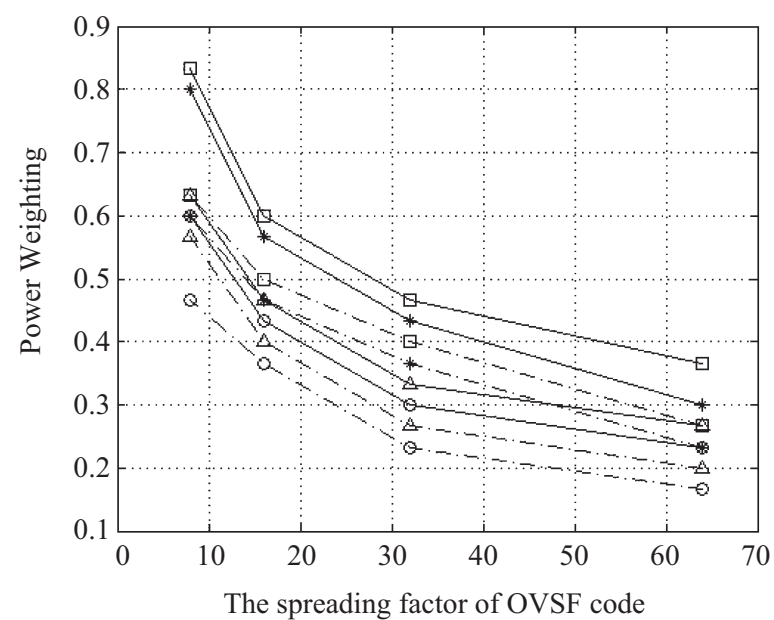

Fig. 4. Transmission power weighting performance of proposed OVSFbased underwater acoustic transmission system with $\mathrm{No}=\mathbf{- 1 0} \mathrm{dB}$. (O, dotted line: 1/3 convolution, BPSK, audio; $\triangle$, dotted line: 1/3 convolution, QPSK, audio; *, dotted line: $1 / 2$ convolution, BPSK, audio; $\square$, dotted line: $1 / 2$ convolution, QPSK, audio; $O$, line: $1 / 3$ convolution, BPSK, image; $\triangle$, line: $1 / 3$ convolution, QPSK, image 0 ; *, line: $1 / 2$ convolution, BPSK, image; $\square$, line: $1 / 2$ convolution, QPSK, image)

audio signals is 0.0033. As shown in the figure, the quality of the audio signal is good. Figure 6 shows the received JPEG2000 image when the power assignment algorithm is used. The peak SNR (PSNR) of the received image signal is $42.8 \mathrm{~dB}$. Figure 7 shows the received JPEG2000 image in the case that the power assignment algorithm is not used. From Figs. 6 and 7, we observe that it is feasible to use the proposed

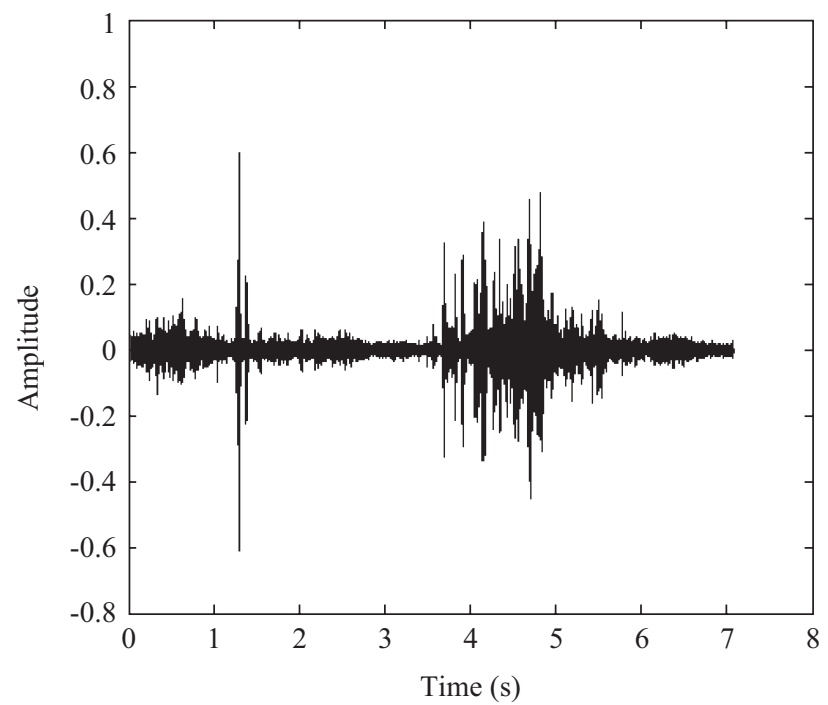

Fig. 5. Received and decoded G.729 audio signals with power assignment mechanism. $(\mathrm{MSE}=\mathbf{0 . 0 0 3 3})$

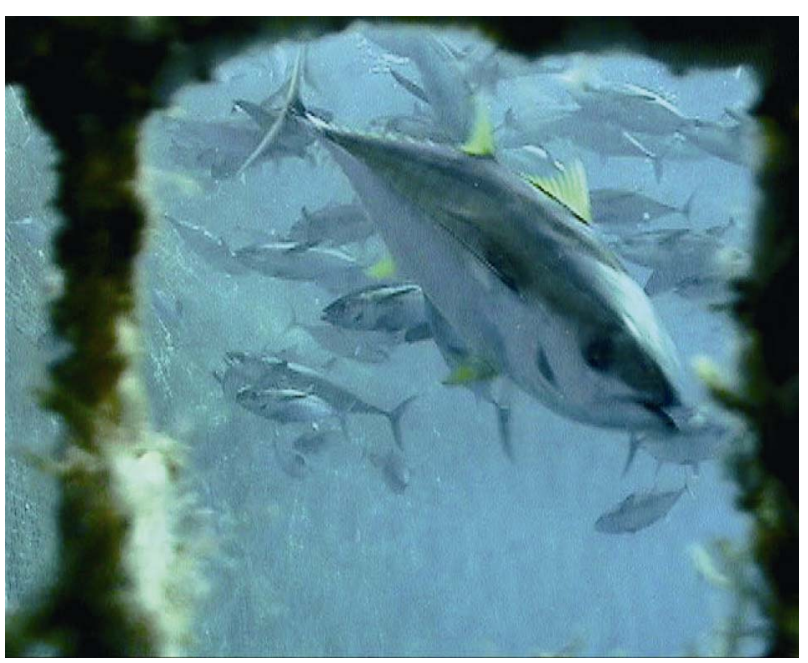

Fig. 6. Received and decoded JPEG2000 image signals using power assignment algorithm. (PSNR = 42.8 dB)

power assignment algorithm is feasible in the underwater acoustic communication system. Further, we can conclude that the proposed system can efficiently transmit audio and image signals.

\section{CONCLUSION}

In this paper, we proposed an underwater acoustic multimedia transmission scheme in which the CSMA/CA protocol, OVSF code, DM transmission strategy, a power assignment algorithm, adaptive modulation, and an unequal error protection scheme were employed. We also performed a simulation to demonstrate the functionality of the proposed system; the simulation results were in good agreement with the theoretical discussion. The proposed system can efficiently 


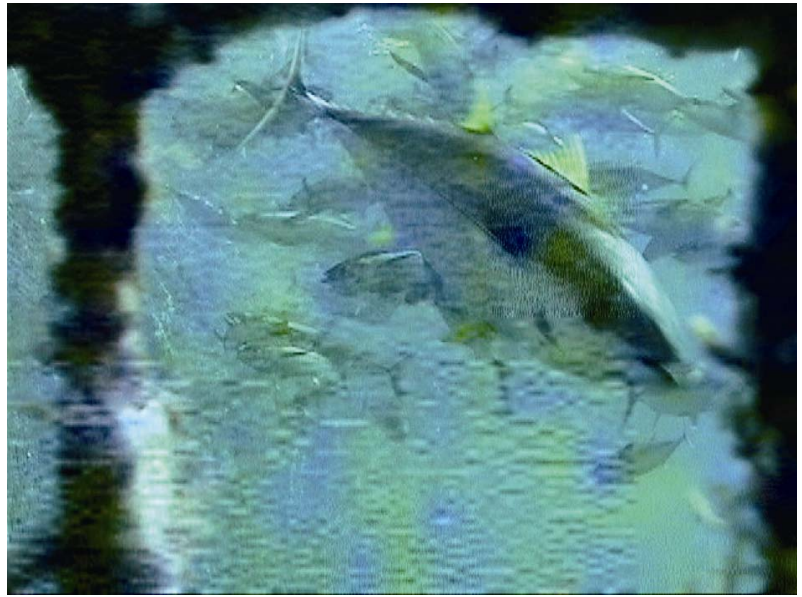

Fig. 7. Received and decoded JPEG2000 image signals without power assignment algorithm. $($ PSNR $=\mathbf{2 4 . 1} \mathrm{dB})$

transmit audio and image signals. In addition, it can achieve maximum transmission data rates or minimum transmission power.

\section{ACKNOWLEDGMENTS}

The authors acknowledge the support of the grant from the National science Council of Taiwan NSC 93-2218-E-019-024 as well as 96-2221-E-002-016 and the valuable comments of the reviewers.

\section{REFERENCES}

1. Baggeroer, A., "A survey of acoustic telemetry," OCEANS, pp. 48-54 (1981).

2. Baggeroer, A., "Acoustic telemetry-An overview," IEEE Journal of Oceanic Engineering, pp. 229-235 (1984).

3. Calvo, E. and Stojanovic, M., "Efficient channel-estimation-based multiuser detection for underwater CDMA systems," IEEE Journal of Oceanic Engineering, pp. 502-512 (2008).

4. Chang, P. R. and Lin, C. F., "Design of spread spectrum multi-code CDMA transport architecture for multimedia services," IEEE Journal on Selected Areas in Communication, pp. 99-111 (2000).

5. Freitag, L., Stojanovic, M., Singh, S., and Johnson, M., "Analysis of channel effects on direct-sequence and frequency-hopped spread-spectrum acoustic communication," IEEE Journal of Oceanic Engineering, pp. 586$593(2001)$

6. Gui, J. H., Kong, J., Gerla, M., and Zhou, S., "The challenges of building scalable mobile underwater wireless sensor networks for aquatic applications," IEEE Network, pp. 12-18 (2006).

7. IEEE P802.11 Wireless LANs, "TGn sync proposed technique specification," IEEE 802.11-04/0889r6 (2005)

8. Kilfoyle, D. B. and Baggeroer, A. B., "The state of the art in underwater acoustic telemetry," IEEE Journal of Oceanic Engineering, pp. 4-26
(2000).

9. Konstantakos, D. P., Tsimenidis, C. C., Adamas, A. E., and Sharif, B. S. "Comparison of DS-CDMA and MC-CDMA techniques for dual-dispersive fading acoustic communication networks," IEE Proceeding Communication, pp.1031-1038 (2005).

10. Lin, C. F. and Chang, K. T.," A power assignment mechanism in ka band OFDM-based multi-satellites mobile telemedicine," Journal of Medical and Biological Engineering, pp. 17-22 (2008).

11. Lin, C. F., Chang, S. H., Chen, J. Y., and Yan, J. T., "A power assignment mechanism for underwater wireless multimedia," Proceeding of MTS/IEEE Ocean (2008).

12. Lin, C. F., Chang, W. T., Lee, H. W., and Hung, S. I., "Downlink power control in multi-code CDMA mobile medicine system," Medical \& Biological Engineering \& Computing, pp. 437-444 (2006).

13. Lin, C. F., Chen, J. Y., Shiu, R. H., and Chang, S. H., "A Ka band WCDMA-based LEO transport architecture in mobile telemedicine," Telemedicine in the 21st Century, edited by Lucia Martinez and Carla Gomez, Nova Science Publishers, pp. 187-201 (2008).

14. Lin, C. F. and Li, C. Y., "A DS UWB transmission system for wireless telemedicine," WSEAS Transactions on Systems, pp. 578-588 (2008).

15. Lin, C. F., Shih, C. H., Chen, C. P., Leu, S. W., Wu, J. K., Tseng, C. H., Hung, H. S., Lu, F. S., Parinov, I. A., and Chang, S. H., "An OFDM-based transmission scheme for underwater acoustic multimedia," WSEAS Transactions on Communications, pp. 343-352 (2009).

16. Pompili, D., Melodia, T., and Akyildiz, I. F., "A CDMA-based medium access control for underwater acoustic sensor networks," IEEE Transactions on Wireless Communications, pp. 1899-1909 (2009).

17. Proakis, J. G., Sozer, E. M., Rice, J. A., and Stojanovic, M., "Shallow water acoustic networks," IEEE Communications Magazine, pp. 114-119 (2001).

18. Rutgers, D. P. and Akyildiz, I. F., "Overview of networking protocols for underwater wireless communications," IEEE Communications Magazine, pp. 97-102 (2009).

19. Sozer, M., Stojanovic, M., and Proakis, J. G., "Underwater acoustic networks," IEEE Journal of Oceanic Engineering, pp. 72-83 (2000).

20. Stojanovic, M., "Recent advances in high-speed underwater acoustic communications," IEEE Journal of Oceanic Engineering, pp. 125-136 (1996).

21. Stojanovic, M. and Freitage, L., "Multichannel detection for wideband underwater acoustic CDMA communication," IEEE Journal of Oceanic Engineering, pp. 685-695 (2006).

22. Technical Specification Group Radio Access Network-Multiplexing and Channel Coding (FDD), 3rd Generation Partnership Project (3GPP), Release 7, pp. 25-212 (2007).

23. Technical Specification Group Radio Access Network-Spreading and Modulation (FDD), 3rd Generation Partnership Project (3GPP), Release 7, pp. 25-213 (2007).

24. Tsimenidis, C. C., Hinton, O. R., Adams, A. E., and Sharif, B. S., "Underwater acoustic receiver employing direct-sequence spread spectrum and spatial diversity combining for shallow-water multiaccess networking," IEEE Journal of Oceanic Engineering, pp. 594-603 (2001).

25. Woo, M., Prabhu, N., and Ghafoor, A., "Dynamic resource allocation for multimedia services in mobile communication environments," IEEE Journal on Selected Areas in Communication, pp. 913-922 (1995).

26. Zhang, J., Zheng, Y. R., and Xiao, C.,'Frequency-domain equalization for single carrier MIMO underwater acoustic communications," Proceeding of MTS/IEEE Ocean (2008). 